\title{
The Arabic Origins of English and Indo-European "Fashion and Modeling Terms": A Radical Linguistic Theory Approach
}

\author{
Zaidan Ali Jassem \\ Department of English Language and Translation \\ Qassim University, KSA \\ zajassems@gmail.com
}

\begin{abstract}
This paper traces the Arabic origins or cognates of English, German, French, Latin, Greek, and Sanskrit "fashion and modeling terms" from a radical linguistic (or lexical root) theory perspective. The data comprises 130 such terms like attire, blouse, chemise, cuff, dress, embroidery, frills, gown, hat, jacket, model, needle, trousers, robe, shirt, shorts, underwear, vest, wool, and so on. The results clearly indicate that all such words have true Arabic cognates with the same or similar forms and meanings, whose differences are due to natural and plausible causes and different routes of linguistic change, especially lexical or semantic shift. Therefore, the results support the adequacy of the radical linguistic theory according to which, unlike the Family Tree Model or Comparative Method, Arabic, English, German, French, Latin, Greek, and Sanskrit not only belong to the same language family, renamed Eurabian or Urban family, but also are dialects of the same language, with Arabic being their origin all because only it shares the whole cognates with them all and because it has a huge phonetic, morphological, grammatical, and lexical variety, wealth, and versatility. On a more general level, they also show that there is a radical language from which all human languages stemmed and which has been preserved almost intact in Arabic, thus being the most conservative and productive language.
\end{abstract}

Keywords : fashion and modeling terms, Arabic, English, German, French, Latin, Greek, Sansk rit, historical linguistics, radical linguistic (lexical root) theory, language relationships

\section{INTRODUCTION}

Jassem (2012a-f, 2013a-q, 2014a-k, 2015a-h, 2016a-b) has demonstrated in forty seven studies so far that Arabic, English, German, French, and the so-called Indo-European languages in general are genetically related very closely phonetically, morphologically, grammatically, and semantically or lexically so much so that that they can all be considered dialects of the same language. More precisely, the Arabic origins or cognates of their words were successfully traced in twenty seven lexical studies in key semantic fields like numerals, religious, love, democratic, military, legal, urban, and floral terms (Jassem 2012a-d, 2013a-q, 2014a-k, 2015a-h, 2016b); in three morphological studies on inflectional and derivational markers (Jassem 2012f, 2013a-b); in nine grammatical papers like pronouns, verb 'to be', wh-questions, and case (Jassem 2012c-e, 20131, 2014c, 2015d); and in one phonetic study about the English, German, French, Latin, and Greek cognates of Arabic back consonants (Jassem 2013c). Furthermore, the theory was extended in another five even wider studies to the examination of the Arabic origins of pronouns in Chinese (Jassem 2014h) and Basque and Finnish (Jassem 2014i), demonstratives (Jassem 2015i), negation (Jassem 2015j), and plurality (Jassem 2016a) in eleven major (and minor) language families in the last three especially, which make up $95 \%$ of the total world population. Finally, two papers applied the approach to translation studies (Jassem 2014e, 2015b).

The above forty seven studies have initially employed the lexical root theory (Jassem 2012a-f, 2013aq, 2014a-g, 2015a-h) and subsequently its slightly revised and extended version, called radical linguistic theory (Jassem $2014 \mathrm{~h}-\mathrm{k}, 2015 \mathrm{a}-\mathrm{j}, 2016 \mathrm{a}-\mathrm{b}$ ), both deriving their name originally from the use of lexical (consonantal) roots or radicals in retracing genetic relationships between words in world languages. The theory first arose as a rejection of the Family Tree Model or Comparative Method in historical linguistics for classifying Arabic as a member of a different language family than English, German, French, Latin, Greek, Sanskrit, and the so-called Indo-European languages (Bergs and 
Brinton 2012; Algeo 2010; Crystal 2010: 302; Yule 2014; Campbell 2004: 190-191; Crowley 1997: 22-25, 110-111; Pyles and Algeo 1993: 61-94). In all the above forty seven studies, the tightly-knit genetic relationship between Arabic and such languages was, on the contrary, categorically established phonetically, morphologically, grammatically, and semantically or lexically so much so that they can be really considered dialects of the same language, where Arabic was found to be their source or parent language for several reasons (Jassem (2012a-f, 2013a-q, 2014a-k, 2015a-g). In other words, Arabic, English, German, and French words of all types and sorts, for example, were shown to be true cognates with similar or identical forms and meanings, whose apparent differences are due to natural and plausible causes and diverse routes of linguistic change. This entails that all such languages developed, in fact must have developed, from an earlier single, perfect, suddenly-emerged Radical Language from which all human languages emanated in the first place, and which could never have died out but rather has fully, though variably, survived into today's languages, to which they can all be traced, with Arabic in particular being the closest or most conservative and productive descendant. To adequately depict the close genetic linkage between Indo-European and Arabian languages in general, a new larger language family grouping has been proposed, called Eurabian or Urban (Jassem 2015c: 41; 2015d).

This paper carries on the quest by investigating the Arabic origins and/or source cognates of fashion and modeling terms in English, German, French, Latin, Greek, Sanskrit, and the so-called IndoEuropean languages. The remainder of the paper has four sections: (ii) research methods, (iii) results, (iv) discussion, and (v) conclusion.

\section{RESEARCH METHODS}

\subsection{The Data}

The data consists of 130 fashion and modeling terms like attire, blouse, chemise, cuff, dress, embroidery, frills, gown, hat, jacket, model, needle, trousers, robe, shirt, shorts, underwear, vest, wool, and so on in English, German, French, Latin, Greek, Sanskrit, and Indo-European languages as well as Arabic, now all generally called Eurabian or Urban. Their selection has been based on the author's knowledge of their frequency and use in today's fully natural English, German, and French conversations and/or texts as well as English dictionaries and thesauri. For ease of reference, the data will be arranged alphabetically together with brief linguistic comments in the next Results section (3).

As for etymological data, all references to English and Indo-European languages are for Harper (2016) despite, like all other similar dictionaries upon which it was based, its severe shortcomings owing to the seemingly illogical, uncertain, or unknown derivations or meanings of many words such as bikini, blouse, cover, curly hair, dress, fashion, fur, furl, model, robe, wool and so on which make more sense if derived straight from Arabic as shall be seen in sections ( $3 \& 4$ ) below. Therefore, it, along with similar dictionaries, has to be used with extreme care and discretion.

Concerning Arabic data, the meanings are for Ibn Manzoor (2013) in the main, Ibn Seedah (1996: 4/63-117), Altha3alibi (2011: 269-275), Albabidi (2011: 229-320), e-dictionaries like mu3jam alama3ani (2016), and the author's knowledge and use of Shami (Syrian) Arabic as a native speaker. All the genetic linkages between Arabic, English, German, French, Latin, Greek, Sanskrit and so on are exclusively mine, unless otherwise stated.

In transcribing the data, normal Romanized spelling is used for all languages for practical purposes. Nonetheless, certain symbols were used for unique Arabic sounds: namely, $/ 2 \& 3 /$ for the voiceless and voiced pharyngeal fricatives respectively, $/ \mathrm{kh} \& \mathrm{gh} /$ for the voiceless and voiced velar fricatives each, /q/ for the voiceless uvular stop, capital letters for the emphatic counterparts of plain consonants /T (t), D (d), Dh (dh), \& S (s)/, and /'/ for the glottal stop (Jassem 2013c). Long vowels in Arabic are usually doubled- i.e., /aa, ee, \& oo/.

\subsection{Data Analysis}

\subsubsection{Theoretical Framework: Radical Linguistic Theory}

Data analysis shall utilize the Radical Linguistic Theory (Jassem 2014h-1, 2015a-j, 2016a-b), a slightly revised and more generalized version of the original Lexical Root Theory (Jassem 2012a-f, 2013a-q, 2014a-g). For the sake of brevity and economy, the inquisitive reader is referred to any 
The Arabic Origins of English and Indo-European "Fashion and Modeling Terms": A Radical Linguistic Theory Approach

earlier work for a fuller account of principles, precepts, and procedures (e.g., Jassem 2015a-c, 2014a, 2013a, 2012a-b).

In short, however, the main principle of the theory states that Arabic is not only related to IndoEuropean languages but also is their immediate ancestor or origin all. In practice, the most appropriate procedure for genetically relating English and Arabic words to each other can be summed up as follows:

- select a word (in any given semantic field), e.g., dress, fashion;

- identify the source, daughter, or sister language meaning (e.g., English or Latin) on the basis of especially word history or etymology. It is essential to start with meanings, not sounds or sound laws as the former are more stable and change a lot less than the latter which do so extensively and drastically; for example, all the sounds of a given word might change beyond recognition while meanings in a rather limited way; so the meaning first will often lead you to the correct cognate naturally whereas the sounds first will get you lost definitely;

- search for the word with the equivalent meaning and form in the target, parent, or reference language (e.g., Arabic), looking for cognates: i.e., sister words with the same or similar forms and meanings;

- explain the differences, if any, in both form and meaning between the cognates lexicologically, phonetically, morphologically, and semantically as indicated. As a matter of fact, finding the right cognate on the basis of its meaning first often leads one to the resultant changes automatically;

- finally, formulate phonological, morphological, grammatical, and semantic rules after sufficient data has been amassed and analyzed.

That is the whole story simply, briefly, and truly. No fuss, no mess. For example, consider attire, dress, fashion, model or any word in Section 3 below.

\subsubsection{Statistical Analysis}

The percentage formula will be used for calculating the ratio of cognate words or shared vocabulary (Cowley 1997: 173, 182), which has been fully described in earlier papers (Jassem 2012a-f, 2013a-q, 2014a-k).

\section{ReSUltS}

The results will primarily focus on the Arabic lexical (consonantal) radicals or roots of English, German, French, Latin, Greek, and Sanskrit fashion and modeling words and the changes that occurred to them. The exact quality of the vowel is, therefore, of generally secondary importance for having little or no semantic impact on the final output whatsoever (Jassem 2012-2016).

Apron (napkin) via Old French naperon 'small table-cloth', diminutive of nappe 'cloth', from Latin mappa 'napkin', from Arabic nabia(tun) 'a palm-leaf meal spread' via lexical shift and turning /t/ into /r/; anbaar 'of food, amounts; a trader's clothing store' via lexical shift and reordering.

Attire (tire, tyre) via Old French atirier 'to equip, ready, prepare; to dress in finery, to adorn', from (i) $a$ - 'to', from Arabic ' $a$ - 'verbal prefix' (Jassem 2013a), and (ii) tire 'order, row, dress', from Arabic ridaa' 'dress' via reversal and turning /d/ into /t/; dithaar 'attire, dress', merging /d \& th/ into /t/; izaar 'dress', turning /z/ into /t/; dir3 'a woman's dress', turning /d \& 3/ into /t \& Ø/; or raiTa(t) 'a womans' dress' via reordering and changing / T/ into /t/.

Bandage (band, bond, bend, bind) via Middle French, from Old French bander 'to bind', from Old High German binda, Sanskrit bandhan 'a tying, bandage', direct from Arabic bandak 'button hole; a piece of cloth' or baneeqa(t) 'a piece of cloth to lengthen a dress' via lexical shift and turning or splitting /q (k)/ into /d (\& j) /; biTaana $(t)$ 'a lining; belly binding' via reordering and turning /T/ into /d/; badana(t) 'a female's sleeveless or pocketless dress' via lexical shift and reordering; or bainaT 'knitter, tailor', banaT (v) via lexical shift and turning /T/ into /t/.

Belt via Old English belt, Old High German balz 'belt, girdle', from Latin balteus 'girdle, sword belt', direct from Arabic ribaaT 'a strap, a tie' via reordering and turning $/ \mathrm{r} \& \mathrm{~T} /$ into $/ 1 \& \mathrm{t} /$.

Bikini via French after Pikinni 'a Marshall Islands atoll in which a US A-bomb test was carried out in 1946', perhaps from (i) pik 'surface', direct from Arabic baqee3, biqaa3 'planted, flat grounds' 
via /3/-loss, and (ii) $n i$ 'coconut. Alternatively, it derives direct from Arabic niqaab 'a female's head and face cover with eye holes; lit., hole, a tear' via lexical shift, reordering, and turning /q/ into $/ \mathrm{k} /$.

Blouse (barn) via French 'peasant's smock', perhaps akin to Provençal lano 'short (wool)', direct from Arabic libas 'dress' via reordering and lexical shift; or bazz 'clothes or such a type' via lexical shift, ///-insertion, and turning /z/ into /s/.

Boot (better, computer booting) via (i) Old French bote 'boot', perhaps from a Germanic source, from Arabic sibtia(t) 'shoes; tanned skin', (spoken Arabic sabbaaT or even booT 'boot') via /s \& b/merger; batt, bathth 'a woolen clothing' via lexical shift (ii) Old English bot 'help, advantage, relieve, atonement; lit., a making better', from Arabic (a) Taab 'to get better' or taab 'to abandon, repent' via reversal and passing /T/ into /t/, and (b) bada 'start (compter)', turning /d/ into /t/.

Bra (brasserie, bracelet) via Old French brassière 'child's chemise, shoulder strap', from Old French braciere 'arm guard', from bras 'an arm', from Latin brachium 'an arm, a forearm', from Greek brakhion 'an arm', from Arabic bark 'breast' via lexical shift and turning / $\mathrm{k} /$ into /s (Ø)/.

Bracelet (brace, embrace, bra) via Old French bracelet, diminutive of bracel, from Latin bracchiale 'armlet', from brachium 'an arm, a forearm', from Greek brakhion 'an arm', from Arabic bark 'breast' via lexical shift and turning / $\mathrm{k} /$ into /s $(\varnothing) /$; or from Arabic raqaba(t) 'neck; a roundshaped object' via lexical shift, reordering, and passing /q/ into /s/.

As to the French and Latin suffixes $-e l(-a l e)$ and $-t$, the first two came direct from Arabic $a l$ - 'the' whereas the last from - at 'fem. suf.'; morphological shift applied to all (Jassem 2012d, 2013a).

Braid via Old English bregdan 'weave, knit, join together; move quickly; vary', Old High German brettan, from Arabic rabaT 'to tie, fold' via reordering and passing /T/ into /d/.

Burnoose straight from Arabic burnus 'burnoose; a dress with head cap'.

Button (butt) via Old French boton 'a button; originally, a bud', from bouter, boter 'to thrust, push', from Arabic biTaana(t) 'a lining' via lexical shift and turning /T/ into /t/; baTTa, baTTa(tun) (n) 'to bulge out' (cf. bottom, butt from Arabic badda 'to have a large backside' via lexical shift).

Cabin (cabinet) via Old French, from Latin capanna 'hut', direct from Arabic khabina(t) 'a room for clothes and trousers', turning $/ \mathrm{kh} /$ into $/ \mathrm{k} /$.

Canvas (canvass, hemp) via Old French chanevaz 'hempen', from Latin cannabis, from Greek kannabis 'hemp', Persian kanab, from Arabic qinnab 'a plant used for ropes', turning /q \& b/ into $/ \mathrm{k} \& \mathrm{v} /$.

Cap (cape) via Old English cappe 'head-covering, hood, cape', from Late Latin cappa 'a cape, hood, cloak', possibly short for capitulare 'headdress', from caput 'head', straight from Arabic $q a b b(a t)$ 'a head's place in a dress', qibaa' 'clothes', qubba3a(t) 'cap; head cover', turning /q \& 3/ into /k \& Ø/ (cf. qahb 'big mountain; cape' via /h/-loss).

Carpet via Old French, from Latin carpita 'thick woolen cloth', probably from carpere 'to card, pluck', direct from Arabic zarabia(t) 'carpet', changing /z/ into /k/.

Cat Walk Show consists of (i) Old English catt, German Katze, from Latin cattus, catta, Byzantine Greek katta, direct from Arabic qiTT(at) 'cat', changing /q \& T/ into /k \& t/ (Jassem 2013g); (ii) Old English wealcan 'to toss, roll, move round', from Arabic walaq 'walk' where /q/ became / $/$ (Jassem 2013n); and (iii) Old English scewaian 'to look at, see', Old Saxon skauwon, Old High German scouwon, from Proto-Germanic root *skau- 'look at', from PIE *skou-, skeue- 'to perceive', from Arabic qasha3 (spoken sha33a) 'to see' via /q \& sh/-merger and /3/-loss; or shaaf 'see', turning /f/ into /w/ (Jassem 2012o).

Chemise (camisole) via Old English cemes 'shirt', from Old French chemise 'shirt, undertunic', Spanish camisa, from Late Latin camisia 'shirt, tunic', probably via Proto-Germanic like German hemd 'shirt', straight from Arabic qameeS 'shirt' or khameeSa(t) 'a shirt', turning /q (kh) $\& \mathrm{~S} /$ into /k (ch) \& s/ (cf. German hemd and Arabic hidm 'dress' via reordering and lexical shift). 
Cloak (cloakroom; clock) via Old French cloque, cloche, cloke 'travelling cloak', from Latin clocca 'traveler's cape; lit., bell', direct from Arabic qaliq 'a kind of mantle' and related qalaqi 'a necklace' and qalaq 'noise' via lexical shift and turning /q/ into /k/; laklook 'socks', laklak, lakka (v) 'to wrap, fold, or tie around' via reordering and lexical shift; silk 'wire, mantle, head cover' via lexical shift and turning /s/ into /k/; khaliq 'of clothes, old' via lexical shift and passing $/ \mathrm{kh}$ \& q/ into /k/ both; khaal 'soft dress', khill/khall 'old dress', khajil 'old dress' via reordering and turning $/ \mathrm{kh} \& \mathrm{j} /$ into $/ \mathrm{k} /$.

Cloth (clothe, clad) via Old English cloth 'a cloth (covering), sail, garment; a woven material to wrap around oneself', German Kleid 'garment, dress', from Arabic kalla(t), kilia(t) 'a thin covering' via lexical shift and turning /t/ into /th/; kiswa(t) 'clothing', merging /s \& $\mathrm{t} /$ into /th/ and inserting $/ \mathrm{l}$; or 2 ullat 'a new, good dress; garment' via lexical shift and turning $/ 2 \& \mathrm{t} /$ into $/ \mathrm{k} \&$ th/.

Coat (waistcoat, coat of arms) via Old French cote 'coat, robe, tunic, overgarment', from Frankish *kotta 'coarse cloth', Old Saxon kot 'woolen mantle', or German Kotze 'a coarse coat', direct from Arabic ghiTaa' 'a cover', turning /gh \& T/ into /k \& t/; qaTeefa(t) 'a kind of coat', replacing /q/ by /k/ and merging / $\mathrm{T} \& \mathrm{f} /$ into /t/; or qadd 'a baby goat's skin' via lexical shift and changing /q \& $\mathrm{d} /$ into $/ \mathrm{k} \& \mathrm{t} /$.

Coat of arms (army, armada, armament, armistice, disarmament) via Old French, from Latin armata 'armed force', from armatus, from armare (v) 'to arm', related to arma 'tools, arms', from Arabic rum2, rimaa 2 (pl.) 'arrow' via /2/-loss; or rami, rumaat (pl.) 'thrower, archer', ramia(t) 'featherless, mud-containing arrow head', marma(t) 'arrow' via reordering and lexical shift (Jassem 2015e).

As to of, it comes straight from Arabic $f i$ 'in, of' or $d h u$ 'possessive of' via reversal and passing /dh/ into /f/ (Jassem 2014c); as for -s, it derives direct from Arabic /t/ 'plural, (also feminine, and derivational) marker' which became /s/ (Jassem 2012f; 2016a).

Collar (blue collar, white collar) via Old French coler (Modern collier) 'neck, collar', from Latin collare 'necklace, band or chain for the neck', from collum 'the neck', from Arabic qilaada(t) 'necklace', turning /q \& d/ into /k \& r/; kaahil 'shoulder and neck area' via lexical shift and /h/loss; or kalkal 'breast, side' via lexical shift and turning /// into /r/.

Comb via Old English and Old High German camb 'comb, crest, honeycomb', German Kamm, Greek gomphos 'a molar tooth', from Arabic qawama 'to straighten, to comb' via lexical shift and /b/split from $/ \mathrm{m} /$; qim3 'a cone' via lexical shift and /3/-loss; kumm 'of flowers, a follicle; tightening' via lexical shift and $/ \mathrm{b} /$-split from $/ \mathrm{m} /$.

Costume (custom, accustom) via French, from Italian costume 'fashion, habit', from Latin consuetudinem, consuetudo (nom.) 'custom, habit, usage', from consuetos, past participle of consuescere 'accustom', from (i) com- 'intensive prefix; together', (Spanish como 'as, like'), from Arabic jamee 3 'together' via /3/-loss and turning /j/ into /k/ or kama 'as, like' (Jassem 2013a) and (ii) suescere 'become used to', related to sui, genitive of suus 'oneself', from Arabic dhu, dhaat 'self; of' where /dh \& t/ became /s/ both. However, it derives more logically, as a whole, from Arabic kasm 'manner, shape, face; body hugging (cloth)' via lexical shift and splitting /t/ into /st/; Taqm 'suit' via reordering, splitting /T/ into /st/, and replacing /q/ by /k/; or hidm, hudoom (pl.) (and related hindaam) 'clothes', turning $/ \mathrm{h} /$ into $/ \mathrm{k} /$ and splitting $/ \mathrm{d} /$ into $/ \mathrm{st} /$.

Cotton via Old French coton, Old Spanish algodon, German Kattun, straight from Arabic quTn 'cotton', turning /q \& T/ into /k \& t/ (Harper 2016).

Cover girl (coverage; discovery; recovery) via Old French covrir (Modern couvrir) 'to cover, protect', from Latin co(o)perire 'to cover over, bury', from (i) com- 'intensive prefix', from Arabic above; and (ii) operire 'to close, cover', straight from Arabic kafar 'to bury, to cover' or ghafaara(t) 'a woman's head cover', turning /gh/ into /k/ (Jassem 2015c).

As to girl, it comes via Old English gyrle 'child of either sex' (with /V being a diminutive insertion), from Arabic ghirr(at) 'a child of either sex', turning /gh/ into /g/; or jaaria(t) 'young girl' where /j/ became /g/ (Jassem 2013k). 
Crown (coronal, coronary, coronation; horn) via Old French corone (Modern coroune), from Latin corona 'crown', from Greek korone 'kind of crown, anything curved', from Arabic qarn 'horn, anything curved' where /q/ became /k/ (Jassem 2015e).

Cuff (handcuff, fisticuffs) via cuffe 'hand covering, mitten, glove; bottom of a sleeve', perhaps via Medieval Latin cuffia 'head covering', direct from Arabic kaff 'palm of the hand; folding back' or koofia(t) '(Palestinian) head covering' via lexical shift; or kumm 'cuff', turning /m/ into /f/.

Curly Hair (curl) probably from an unrecorded Old English word or Dutch krul 'curly', from ProtoGermanic *krusl- (East Frisian krull 'lock of hair', Middle High German krol, direct from Arabic ghurra(t) 'a frontal lock of hair', turning /gh/ into /k/; kawar 'to circle' or kar(kar) 'to turn', splitting // from /r/; lakka 'to curl, to round' via reversal and /r/-split from ///; or khiSla(t) 'a lock of hair', merging /kh \& S/ into /k/ and splitting /r/ from /1/. See hair.

Dress (address, redress) via Old French dresser, drecier 'raise (oneself), address, prepare, set (a table), serve (food), straighten, direct', from Vulgar Latin directiare, from Latin directus 'direct, straight', direct from Arabic rids/dars '(old) clothes; lesson' via reordering; daraz 'to sew' or Taraz 'to embroider' via lexical shift and passing /T \& z/ into /d \& s/; or dithaar 'a dress' via reordering and changing $/$ th/ into $/ \mathrm{s} /$.

Embroidery (embroider) via Anglo-French enbrouder, from (i) en- 'in', from Arabic in- 'verbal affix' (Jassem 2013a) and (ii) broisder 'embroider', from Frankish *brozdon, from Proto-Germanic *bruzdajan, direct from Arabic burda(t) 'a strapped dress' via lexical shift (cf. duroob 'lines, straps', mudarrab (adj.) 'of clothes, strapped' via reordering).

Fabric (fabrication) via Middle French fabrique, from Latin fabricare 'to make, construct, fashion, build', from fabrica 'workshop, an art, trade, fabric', from faber 'artisan who works in hard materials', from Arabic farw 'fur', farwati (n) 'fur maker' via reordering and turning $/ \mathrm{w} /$ into $/ \mathrm{b} /$; zubar/wabar 'tiny wool, hair; cutting' through lexical shift and passing /z (w)/ into /f/; or farak 'to twist, spin, rub; hate' via lexical shift and inserting /b/ or splitting it from /f/.

Fashion (fashionable) via Old French façon, fachon, fazon 'face, appearance; construction, design; thing done; beauty; manner', from Latin factio(nem), factio (nom.) 'a making, doing; a preparing', facere 'to make', direct from Arabic fishshash(un) (fishfash, fashoosh) 'a coarse or soft dress', fasha(sha) (v) 'to spread' via lexical shift (and/n/-insertion); wajh(un), wajjah (v) 'face, mask; manner' where /w \& j/ became /f \& sh/; or faSSal 'to design (clothes)' via lexical shift and turning /S \& $1 /$ into /sh \& $\mathrm{n} /$.

Fatigues (fatigue dress) via French fatigue 'weariness', from Latin fatigare 'to tire out, to weary', from Arabic fataq 'of clothes, to rip, rupture' via lexical shift and turning /q/ into /g/.

Fibre (fibrous, fibrosis, fibril) via Medieval Latin fibre, from Latin fibra 'a fibre, filament; entrails', perhaps related to Latin filum 'a thread, string', from Arabic zi'bar, zubar (pl.) 'tiny wool, hair; cutting' or wabar 'wool', turning /z (w)/ into /f/; or from Arabic farw 'fur' via reordering and turning $/ \mathrm{w} /$ into $/ \mathrm{b} /$. See fur \& fabric.

Fit (outfit, fitting room) perhaps from Old English fit 'an adversary of equal power' or fitt 'conflict, struggle', from Arabic fata, fatee 'a youth; young and strong'; faTTa(t) 'sudden attack of anger; jump', turning /D (T)/ into /t/ (Jassem 2015); however, as a fashion term, from faat 'to enter, to suit'; fiDi 'to suit freely; large' where /D/ became /t/; or waata 'to fit, suit', turning /w/ into /f/.

Fleece (fleecy; floss) via Old English fleos, flies 'fleece, wool, fur', Middle High German vlius, German Vlies, probably from PIE *pleus- 'to pluck; also a feather, fleece', Latin pluma 'feather, down', from Arabic Soof, al-Soof 'wool, the-wool' via reversal or reordering (and ///-insertion); or fatl 'a thin and twisted string or thread; turning' via reordering and turning $/ \mathrm{t} /$ into $/ \mathrm{s} /$.

Frill (frills, frilly, frillery) 'wavy ornamental edging' is of uncertain origin, direct from Arabic rafala 'of clothes, to hang down loose, move or shake around legs' via reordering and lexical shift. See furl.

Fur probably via Old French forrer, fourrer 'cover with fur, line (clothing)', from fuerre 'sheath', from Frankish *fodr or Germanic (Old High German fotar (Modern Futter) 'a lining, sheath'), from PIE root *pa- 'to feed, protect', direct from Arabic farw(at), firaa' 'fur'.

Furl (unfurl) possibly via Middle French ferler 'to furl', from Old French ferliier 'chain, tie up, lock away', perhaps from fer 'firm', from Latin firmus 'firm' + -lier 'to bind', from ligare 'to bind', or 
from furdle 'to furl, fold', direct from Arabic farra, falla 'to unfurl, untie, unloosen' via lexical divergence and /l (r)/-insertion; laffa 'to fold' via reversal and /r/-insertion. See frill.

Gaiter 'leather cover for the ankle' via French guetre 'belonging to peasant attire', perhaps from Middle French *guestre, from Frankish *wrist 'instep', from Proto-Germanic wristiz (German Rist 'instep'), from Arabic ghutra(t) 'head cover; a covering' via lexical shift and turning /gh/ into /g/; or qiTria(t) 'a type of attire' via lexical shift and passing /q \& T/ into /g \& t/.

Garment (undergarment, garnish) via Old French garnement 'garment, clothes, attire', from garnir 'fit out, provide, adorn', direct from Arabic qiraam 'a cover' or khimar 'a woman's head covering' via lexical shift, reordering, and turning /(q) kh/ into /g/.

Girdle (gird, girth) via Old English gyrdel, gyrdan (v) 'belt, cord', Old High German gurtil, German Gürtel 'belt, girdle', direct from Arabic 'iqleed 'a strap, string, rope', qilaada(t) 'necklace', qalad (v) 'to twist, wrap, girdle' via lexical shift, turning /q/ into /g/, and splitting /// into /r \& l; or qurT 'a cloth worn by women', passing /q \& $\mathrm{T} /$ into /g \& d/ and inserting /// besides lexical shift.

Glove via Old English glof 'glove, hand covering; palm of the hand', from Proto-Germanic *galofo 'covering for the hand', probably from (i) $g a$ - 'collective prefix' and (ii) *lofi 'hand', direct from Arabic kaff, al-kaff 'palm of the hand; glove' via reordering and turning /k/ into/g/; or ghilaf 'a covering', turning /gh/ into /g/.

Gown (nightgown) via Old French goune 'robe, coat, (nun's) habit', related to Latin gunna 'leather garment, skin, hide', from Arabic qinaa3 'a woman's (head) dress; cover', jinaan 'a dress', or qinaan 'a shirt's cuff' via lexical shift and turning /q (j) \& $3 /$ into /g \& Ø/.

As to night, it comes from Arabic jinna(t) 'darkness' via lexical shift, reordering, and turning /j/ into /g (Ø)/ (Jassem 2013j).

Guise (disguise) via Old French guise 'manner, fashion, way', from Frankish *wisa or Old High German wisa 'manner, wise', from PIE *weid- 'to see', direct from Arabic ghishaa', ghishawa(t) 'a thin cover; darkness' or kisaa' 'clothing', turning /gh (k) \& sh/ into/g \& s/ (cf. Arabic waDa2 'appear; white', turning /D \& 2/ into /d \& Ø/).

Hair (hairy, hair-shirt) via Old English haer 'hair, a hair', from Proto-Germanic *kharan, German Haar, perhaps from PIE *ghers- 'to stand out, to bristle', direct from Arabic sha3r 'hair', merging /sh \& 3/ into /h/ (Jassem 2013h). See curly.

Hat (hood) via Old English hat 'hat, head covering', from Proto-Germanic *hattuz 'hat, cowl', from PIE root *kadh- 'cover, protect', (Latin cassis 'helmet'), direct from Arabic $2 a T T a(t)$ 'head cover; cap', turning $/ 2 \& \mathrm{~T} /$ into $/ \mathrm{h} \& \mathrm{t} /$; or 2 awtakia(t) 'a hood, helmet', turning $/ 2 /$ into $/ \mathrm{h} / \mathrm{h}$ and merging $/ \mathrm{t} \& \mathrm{k} /$. See hood.

Helmet (helm) via perhaps Middle English helm 'protection, covering, crown, helmet', from French helmet, diminutive of helm (Modern heaume) 'helmet', German Helm, from PIE root *kel- 'to cover, hide', from Arabic la'mat 'battle head gear' via reordering and turning /'/ into /h/; lu2mat 'a soldier's underwear; connection; cover' via reordering and turning $/ 2 /$ into $/ \mathrm{h} /$; haamat, alhaamat '(top of) head' via lexical shift and reordering; or 3imma(t), 3amamat 'head cover; cap', turning $/ 3 \& \mathrm{~m} /$ into $/ \mathrm{h} \& 1 /$.

High Heels via Old English (i) heh, heah 'high, tall', from Proto-Germanic (Swedish hög, German hoch), from Arabic shahiq 'very high' via /sh \& h/-merger into /h/ and turning /q/ into /g (h, Ø)/ and (ii) Old English hela 'back of the foot', Dutch hiel, from PIE *kenk 'bend of the knee, heel', direct from Arabic kaa2il 'ankle' via lexical shift and merging /k \& 2/ into /h/; 3aali 'high' via lexical shift and turning /3/ into /h/; or khulkhaal 'woman's shoes; foot ring or bracelet' via lexical shift, syllable merger, and turning $/ \mathrm{kh} /$ into $/ \mathrm{h} /$.

Hood (hat) via Old English hod 'a hood, soft head covering', from Proto-Germanic *hodaz 'hat, cowl', (German Hut 'hat'), from PIE root *kadh- 'cover, protect', (Latin cassis 'helmet'), direct from Arabic $2 a T T a(t)$ 'head cover; cap', turning $/ 2 \& \mathrm{~T} /$ into $/ \mathrm{h} \& \mathrm{~d} /$. See hat. 
Hoop (hop) probably from Old English *hop 'circular band, flattened ring', Old Frisian hop 'hoop, a band', from Arabic $2 a b l$ 'rope, cable' where $/ 2 \& 1 /$ became $/ \mathrm{h} \& \emptyset /$ or haab 'to walk on one leg' (Jassem 2013n).

Jacket (Jack, jackass) via Old French (i) jaquet 'short coat with sleeves', from jaque (dim.) 'a kind of tunic', or (ii) Jacque 'a male proper name', from Arabic ja2sh 'chief; donkey; proper name' via $12 \& \mathrm{sh} /$-merger into /k/ (Jassem 2014f) or (iii) jaque (de mailles) 'short, tight-fitting coat; coat of mail', a shortening from Spanish iaco, from Arabic shakk 'breastplate' via lexical shift and turning /sh/ into /k/ (Harper 2016), or shuqqa(t) 'of clothes, a cutting or piece' where /sh \& q/ became $/ \mathrm{j} \& \mathrm{k} /$ besides lexical shift.

Jersey means 'a type of knitted cloth; a breed of cattle', both from Jersey 'one of the Channel islands', perhaps a corruption of its Latin name Caesarea, from Arabic jazeera(t) 'island' via reordering and/or jazoor 'a camel', jazar'sheep, goat' via reordering and lexical shift (cf. jaraza, jirza(t) (n) 'to fold or tie something like a bundle of parsley or wheat').

Jewel (jewelry) via Old French, perhaps from Latin jocale, from jocus 'pastime, sport', or from Latin gaudium 'rejoice', direct from Arabic jawhar(at) 'jewel' via /h/-loss and passing /r/ into /l/.

Knit via Old English cnyttan 'to tie with a knot, bind', cnotta 'a knot', Middle Low German knütten 'to tie, knot', direct from Arabic naqad, najad 'to knit or sew (quilts, wool, cotton)' via reordering and turning /q (j) \& d/ into / $\&$ t/.

Leather (leathery, leatherneck) via Old English ledher 'hide, skin, leather', from Proto-Germanic *lethran (German Leder), from PIE *letro- 'leather', direct from Arabic wathir 'a leather worn by menstruating women' or wadhir 'a boneless piece of meat' via lexical shift and passing /w/ into $/ \mathrm{l}$; or jild(at) 'skin, leather' via reordering, merging $/ \mathrm{j} \& \mathrm{l}$, and turning $/ \mathrm{d} \& \mathrm{t} /$ into $/$ th $\& \mathrm{r} /$.

Manicure via French, from Latin (i) manus 'hand', from Arabic 'anamil 'fingers' via lexical shift, reordering, and merging $/ n \& 1 /$ and (ii) cura 'care, concern, trouble', from Arabic riqqa(t) 'carefulness, gentleness' via reversal and changing /q/ into $/ \mathrm{k} /$.

Mantle (dismantle) via Old English mentel 'loose, sleeveless cloak', German Mantel, Old French mantel (Modern manteau), from Latin mantellum 'cloak', direct from Arabic mandeel 'a head cloth; napkin' via lexical shift and turning /d/ into /t/; or malaa'a(t) 'a woman's dress' via reordering and $/ \mathrm{n} /$-insertion.

Mark via Old English mearcian 'to trace out boundaries' and German merken, from Arabic marqoom 'marked', raqam (n) via reordering and passing /q/ into /k/; or makar (also maghar, maragh) 'to paint-mark' via reordering and replacing /gh/ by /k/ (Jassem 2014g).

Mat via Old English matte, from Late Latin matta 'mat made of rushes', from Arabic madda(t) 'mat', passing /d/ into /t/; or mahd 'bed', merging / $\mathrm{h} \& \mathrm{~d} /$ into /t/.

Mattress via Old French materas, from Italian, from Latin matracium, from Arabic al-matrah 'the cushion' (Harper 2016); or from mafrash 'mat, mattress', passing /f \& sh/ into /t \& s/.

Mirror via Old French, from Vulgar Latin *mirare 'to look at', from mirari 'to wonder at, admire', direct from Arabic mir'aa(t) 'mirror', $r a^{\prime} a(\mathrm{v})$ 'see'; /t/ changed into /r/.

Model (modeling, supermodel, mode, modal, accommodate) via Middle French modelle, from Italian modello 'a model, mold', from Vulgar Latin modellus, from Latin modulus 'standard, a small measure', diminutive of modus 'manner, measure', direct from Arabic mathal, mithaal 'likeness, example, standard', turning /th/ into /d/; or mada 'extent, distance, measure', with -el being derived from Arabic - al 'the' via morphological shift (Jassem 2012d, 2013a).

As for supe rmodel, it comes direct from Arabic Subar 'high' via /S/-mutation into /s/ (Jassem 2014c); see overalls.

Necklace consists of (i) neck, from Old English hnecca 'neck, nape', Old High German hnach (Modern Nacken), direct from Arabic 3unuq 'neck' where $/ 3$ \& q/ became /h (Ø) \& k/, and (ii) lace, from Old French laz 'a net, cord, snare', from Vulgar Latin *lacium, from Latin laqueum, laqueus (nom.) 'snare, noose', direct from Arabic 3alaq 'hang, entangle, ensnare' via /3/-loss and replacing /q/ by /s/. See shoe. 
Necktie is from (i) neck above and (ii) tie, from Old English teag 'cord, band, thong, fetter', direct from Arabic Tawq 'band, fetter, brace; a folding' or Tai 'tying, folding', Tawa (v) 'to tie', turning $/ \mathrm{T} \& \mathrm{q} /$ into $/ \mathrm{t} \& \mathrm{~g}(\varnothing) /$.

Needle via Old English naedl, Gothic nethla 'needle', German Nadel, from PIE *net-la, from root *(s)ne-, Greek nein 'to spin', Latin nere 'to spin', direct from Arabic naSl, nasl, naDl 'arrow, needle' via lexical shift and turning /D $(\mathrm{S}, \mathrm{s}) /$ into /d/; or nawl 'a tailor's log for cloth rolls' via lexical shift and /d/-insertion.

Net (nexus) via Old English net 'netting, network, spider web, mesh used for capturing', Old High German nezzi, German Netz, from PIE *ned- 'to tie, bind, twist, knot', Sanskrit nahyati 'binds, ties', Latin nodus 'knot', from Arabic naseej, nasj 'knitting, weaving, binding together' via /s \& $\mathrm{j}$-merger into /t (ks)/. See nexus.

Nexus (net, annex, connection) from Latin nexus 'that which ties or bonds together', from nectere (v) 'to bind', from PIE *ned- 'to tie, bind, twist, knot', from Arabic naseej, nasaj (v) 'knitting; binding together' via /s \& j/-mutation into /k \& s/; or naqad/najad 'to knit, to stitch', turning /q (j) $\& \mathrm{~d} /$ into $/ \mathrm{k} \& \mathrm{~s} /$. See net.

Overalls via Old English ofer eall, from (i) ofer 'beyond, above, upon, in, across, past, on high', German über, from PIE *uper/super 'super', direct from Arabic Subar 'high' via /S/-loss or mutation into /s/, 3abra 'over, across' via /3/-loss and turning /b/ into /v/, or waraa' 'beyond' by replacing $/ \mathrm{w} /$ by $/ \mathrm{v} /$ and (ii) eall 'all, every, entire', from agelik 'all', Old High German al, Gothic alls, from Arabic kull 'all' via /g \& k/-merger into /ea (a)/; or al 'the' or 'ulaa' 'these' via lexical shift.

As to the plural marker $-\mathbf{s}$, it comes direct from Arabic $-t$ 'plural, inflectional, and derivational marker' (Jassem 2012f, 2013a, 2016a)

Pajamas via Hindi pajama, probably from Persian paejamah 'leg clothing, from (i) pae 'leg', from PIE *ped- 'foot', direct from Arabic $i b T$ 'armpit' via lexical shift and turning /T/ into /d/ or boo3 'wrist bone' via /3/-loss and lexical shift (Jassem 2013h) and (ii) jamah 'clothing', from Arabic khaam(at/h) 'cloth' or qimash 'cloth', turning /q (kh) \& sh/ into /j \& h/. Otherwise, as a whole, from Arabic jubba(t) 'a dress' via reordering and turning $/ \mathrm{t} /$ into $/ \mathrm{m} /$; jilbaab 'a woman's dress' via lexical shift, reordering, and turning $/ / /$ into $/ \mathrm{m} /$.

Pantaloon (pants, panty) via Old French 'kind of tights', from Italian (San) Pantalone 'a Christian martyr or saint', from Greek for 'entirely lion; all-compassionate', direct from Arabic tabaan 'short trousers for covering genitalia' via reordering; or bainaT 'knitter, tailor', banaT (v) via lexical shift and turning /T/ into /t/ (cf. Harper 2016).

Pants short for pantaloon above.

Plume via Old French, from Latin pluma 'feather; down; the first beard', direct from Arabic bailam 'cane cotton; lip fetter', balam (v) via lexical shift.

Pullover is a compound of (i) pull, from Old English pullian 'to pluck off (wool), draw out', perhaps related to German pollen 'remove the shell or husk', direct from Arabic labeeba(t) (n) 'a sleeveless and pocketless dress', labbab (talabbaba) (v) 'to roll up clothes and tie them around the waist' via reordering and lexical shift; or libs 'dress' via reordering and deleting /s/; and (ii) over as in overalls above.

Purse (bursary, bourse) via Old English pursa 'little leather bag', from Latin bursa, byrsa 'leather bag' (Old French borse, Modern bourse), Greek byrsa 'hide, leather', from Arabic qirba(t) 'a leather bag' or jirab 'a bag, purse' via reversal and turning /q (j)/ into /s/.

Ring (earring, nose ring) via Old English and Old High German hring (German Ring) 'small circlet for wearing on the finger; anything circular', from Proto-Germanic *khrengan, from PIE root * $(s)$ kregh, from (s)ker- 'to turn, bend' (Latin curvus 'bent, curved', crispus 'curly', and perhaps Greek kirkos 'ring', koronos 'curved', direct from Arabic qarn 'a horn or horn-shaped object' or naqar 'to beat, knock' via reordering and turning /q/ into /g/. 
As for ear, it derives from Arabic 3air 'ear' via /3/-loss (Jassem 2013h, 2015c) (cf. air, from Arabic 'air ('iar, 'uiar) 'air' (Jassem 2013e); area, from Arabic 3araa' 'open space' via /3/-loss (Jassem 2013f); eros (erotic), from Arabic 'air 'penis' (Jassem 2013q); and era, from Arabic $3 a S r$ 'time, era' via /3/-loss and /S \& r/-merger (Jassem 2013j)).

2015c).

Concerning nose, it comes from Arabic 'anf 'nose', passing /f/ into /s/ (Jassem 2013h,

Rip probably via Flemish rippen 'strip off roughly', Frisian/Danish rippe 'to tear, rip', from PIE *reup, *reub- 'to snatch', from Arabic $r a$ 'ab or 'abar 'to repair, to mend' via lexical divergence. (Cf. pure from Arabic buhaar, baahir 'white, brilliant' via/h/-loss; and rib from Arabic 'irb 'organ' and 'arib 'part of a finger' via lexical shift.)

Robe (bathrobe, wardrobe; rope) via Old French robe 'long, loose, outer garment', from Germanic (Old High German rouba 'vestments', German roub 'robbery', direct from Arabic thawb 'inner or outer garment', turning /th/ into /r/; or $r u$ 'ba(t) 'a piece of cloth for mending saddles' via lexical shift.

Rope via Old English rap 'rope, cord, cable', from Dutch reep 'rope', Old High German and German reif 'ring, hoop', German roub 'robbery', direct from Arabic ribaaba(t) 'a string or cloth for holding arrows' via lexical shift and syllable merger; or $s a b b$ 'rope, cable', turning /s/ into /r/.

Rug (rugged, rag) probably from Old Norse rögg 'shaggy tuft' or from Danish rag, from ProtoGermanic *rawwa-, perhaps related to rag and rough, direct from Arabic ruq3a(t), riqaa3 (pl.) 'cloth, rug, patch; rugs' via /3/-loss and turning /q/ into /g/; or raqq 'a thin, flat stone or rock', raqeeq (adj.) 'thin' via lexical shift.

Sandal via Old French sandale, from Latin sandalium 'a slipper, sandal', from Greek sandalion, diminutive of sandalon 'sandal', from Arabic sandal 'socks; slippers' via lexical shift.

Seam via Old English seam 'seam, junction', from Proto-Germanic *saumaz (Old High German soum, German Saum 'hem'), from PIE * syu- 'to sew, bind' (Sanskrit syuman 'sew', Latin suere 'sew', Old English siwian, Greek hymen 'thin skin', hymnos 'song', Russian švec 'tailor'), from Arabic Samma 'to close or join tight', samm 'needle hole', or kumm 'cuff', kamma (v) 'to fold back; tie' via lexical shift and turning /S (k)/ into /s/. See sew.

Sew (sewer) via Old English siwian 'to stitch, sew, mend, patch, knit together', from Proto-Germanic *siwjanan (Old High German siuwan 'sew'), from PIE *syu- 'to bind, sew' (Sanskrit sivyati 'sews', Latin suere 'sew', Greek hymen 'thin skin', hymnos 'song', Russian švec 'tailor'), from Arabic sa'wa 'of clothes, to cut' or sawwa 'to straighten, fashion, level, make' via lexical shift. See seam.

Shirt (short, shear) via Old English scyrte 'skirt, tunic', from German Schurtz 'apron', related to Old English scort, sceort 'short', from PIE * (s)ker- 'to cut', from Arabic sutra(t) 'a covering', satar (v) 'to cover' via lexical shift and turning /s/ into /sh/; or sharT 'a string; a piece of cloth; a cutting' via lexical shift and changing $/ T /$ into /t/. See shorts.

Shoe (shoelace, horseshoe) via Old English scoh 'shoe', Swedish sko, German Schuh, from Arabic shis 3 'upper or lower part of shoe' via lexical shift, merging /sh \& s/, and deleting /3/; or 2idhaa' 'shoes', merging $/ 2 \& \mathrm{dh} /$ into $/ \mathrm{sh} /$.

As to lace, it comes from Arabic 'asala(t) 'shoe's pointed head' via reversal and lexical shift. As for horse, it derives from Arabic faras 'horse', passing /f/ into /h/ (Jassem 2013g). See necklace.

Shorts (short) via Old English scort, sceort 'short', probably from Proto-Germanic *skurta- (Old High German scurz 'short'), from PIE *(s)ker- 'to cut' (Latin curtus 'short'), from Arabic qaSeer(at) 'short' or qarT 'cut', merging /q \& $\mathrm{S}(\mathrm{T}) /$ into /sh/.

Silk via Old English seoloc, sioloc 'silk, silken cloth', from Latin sericum 'silk', from Greek Serikos 'silken; pertaining to Seres 'an oriental people from whom the Greeks got silks', perhaps from Chinese si 'silk', Mongolian sirkek, Manchurian sirghe, direct from Arabic saraq 'silk', turning $/ \mathrm{r} \& \mathrm{q} /$ into /l \& k/ (cf. sharqi 'Eastern' or sharaq 'a red dye' via lexical shift and turning /sh, q, $\& \mathrm{r} /$ in $/ \mathrm{s}, \mathrm{k}, \& \mathrm{l} /$; or silk 'head cover; wire, thread' via lexical shift). 
Skirt (miniskirt) via Old Norse skyrta 'shirt, a kind of kirtle', from the same origin as shirt \& shorts above; or from Arabic qurT or kharraaTa(t) 'a cloth worn by women', splitting /q (kh)/ into /sk/ and changing $/ \mathrm{T} /$ into $/ \mathrm{t} /$.

As to miniskirt, it is short for miniature, from Latin minimum (minimize, minute, diminish, diminutive; minus, minor, minority; minister, administer, administration; mono) 'smallest, least; weak', from Arabic maneen (mamnoon), manna (v) 'reduced, lessened', min 'from, minus'; 'umam 'little' or 'amam 'small', turning /m/ into /n/; numnum, nama' 'very small' via reordering; ma3n 'small, little' via /3/-loss (Jassem 2014g)

Skin via Old Norse skinn 'animal hide, fur', Old English scinn, German Schind 'skin of a fruit', from PIE *sken- 'to cut off', from root sek- 'to cut', direct from Arabic shaqq(un), shaqqa (v) 'to cut', turning /sh \& q/ into /s \& k/; otherwise, from Arabic sakina(t) 'head position in the neck' via lexical shift; sa2na(t), su2na(t), sa2naa' 'facial skin or colour' via lexical shift and turning /2/ into $/ \mathrm{k} /$; or salkh 'skin; removing skin' via reordering and turning $/ 1 \& \mathrm{kh} /$ into $/ \mathrm{n} \& \mathrm{k} /$.

Sleeve via Old English sliefe 'arm-covering part of a garment; lit., that into which the arm slips', sliefan (v) 'to slip on (clothes)', slupan 'to slip, glide', from Old High German sloufen 'to put on or off', from Arabic labisa 'to put on' via lexical shift, reordering, and turning /b/ into /f/; or sulfa(t) 'shoe leather lining' and related salf 'big bag' via lexical shift.

Slippers (slip, slippery) 'type of loose, light indoor footwear; a shoe slipped onto the foot', via Old English slypscoh 'slipper', from Middle Low German slippen 'to glide, slip', Old High German slifan, German schleifen 'to glide, slide, from Arabic labisa 'to put on' via lexical shift and reordering; sal2ab, sal2af, zal2af 'to creep' via /2/-loss and lexical shift.

Socks via Old English socc 'slipper, light shoe', Old High German soc, German Socke, from Latin soccus 'slipper', probably from Greek sykchos 'a kind of shoe', from Arabic shikk 'a cloth worn over shoes' via lexical shift and turning /sh/ into /s/; or ziqq 'a leather bag' via lexical shift and turning $/ \mathrm{z} \& \mathrm{q} /$ into $/ \mathrm{s} \& \mathrm{k} /$.

Sole via Old French sole, from Latin sola. solea 'sandal, bottom of a shoe, a flat fish', from solum 'bottom, ground, foundation, lowest point of a thing', from Arabic 'asfal (saafil, sufli) 'low' via lexical shift and /s \& f/-merger; or Silaal 'shoe lining or leg' via lexical shift and passing /S/ into $/ \mathrm{s} /$.

Spin (spinster) via Old English spinnan 'draw out and twist fibers into thread', German spinnen, (cognate with Greek patos 'garment; lit., that which is spun'), from Arabic zaban 'to tie up back; of camels, to push back', zaboon 'a long garment; customer; pusher' via lexical shift and turning $/ \mathrm{z} /$ into /s/; nabaz 'to appear suddenly; to rise' via reordering and lexical shift; or khaban 'to shorten clothes by folding' via lexical shift and turning $/ \mathrm{kh} /$ into $/ \mathrm{s} /$.

Stitch (stick) via Old English stice 'a prick, puncture, sting, stab', from Proto-Germanic *stikiz (source of Old High German stih, German Stich 'prick, stab'), from PEI *stig-i-, from root *steig- 'to stick, pointed', from Arabic shawk(at) 'a prick', shaak, ishtaaka (v) via reordering and turning /sh \& $\mathrm{k} /$ into $/ \mathrm{s} \& \mathrm{ch} /$.

Stockings (stock) 'close-fitting garment covering the foot and lower leg', from stock 'leg covering', from Old English stocu 'sleeve', related to Old English stocc 'trunk, log', Old Norse stuka, Old High German stuhha, direct from Arabic saaq/sooq 'leg, stem' via lexical shift, splitting /s/ into $/ \mathrm{st} /$, and turning /q/ into $/ \mathrm{k} /$.

Strap via Old French estrop 'strap', from Latin stroppus, from Greek strophos 'a twisted band; cord, rope', direct from Arabic ribq(at) 'cable, string', irtabaq, rabaqa (v) 'to tie or tighten with a rope' via reordering and /q/-split into /st/; or karab 'to tighten' via / $/$ /-split into /st/ and lexical shift.

String via Old English streng 'line, cord, thread, string of a bow or harp', from Old High German strang, German Strang 'rope, cord', from PIE strenk- 'tight, narrow', from Arabic sharnaq 'of clothes, torn; cut' or sharak 'rope' via lexical shift, splitting /sh/ into /st/, and passing /q/ into /g/; shareeT, shurTaan (pl.) 'string' via reordering, changing /sh \& T/ into /s \& t/, and inserting /g/; 
or shaTan 'long rope', turning /sh/ into /s/, splitting /T/ into /tr/, and inserting /g/; or zardaq 'string' via reordering, passing /z, d, \& q/ into /s, t, \& g/, and inserting /n/.

Striptease via (i) Old English - striepan, strypan 'to plunder, despoil', German streifen 'strip off, roam', from Arabic Sarab 'cut; earn' via lexical shift and /S/-split into /st/ and (ii) Old English teesan 'pull, pluck, tear, comb', Old High German zeisan 'to tease, pick wool', from Arabic jazza 'to cut wool', turning /j/ into /t/; or shadda 'to pull, pluck (wool)' via reversal and passing /sh \& $\mathrm{d} /$ into $/ \mathrm{s} \& \mathrm{t} /$.

Suit (swimsuit, suitcase, suitable; suitor) via Old French suite, sieute 'pursuit, following, hunt; assembly', from Vulgar Latin *sequita, from sequi (v) 'to follow, attend', from Arabic saaqa 'to drive, follow', turning /q/ into /t/; Said 'hunting', passing /S \& d/ into /s \& t/.; otherwise, direct from Arabic 'aSda(t), 'aSeeda(t), mu'Sada(t) 'a small shirt or underwear for children' via lexical shift and turning /S \& d/ into /s \& t/; or saq3 'any dress', turning /q \& 3/ into /t \& Ø/.

Suspenders (depend, independent) via Old French, from Latin suspendere 'to hang up, kill by hanging; make uncertain; interrupt, stop', from (i) sub- 'up from under', from Arabic Sawb 'falling; towards' via lexical shift (Jassem 2014c) and (ii) pendere 'cause to hang, weigh', from Arabic madd 'extend, stretch' where $/ \mathrm{m} / \mathrm{split}$ into $/ \mathrm{p} \& \mathrm{n} /$, banood(at) 'anus; hanging down', or baTan (v) 'to take into or put around belly' via lexical shift and turning /T/ into /d/ (Jassem 2015e).

Sweater (sweat) via Old English swat 'perspiration; labour, that which causes sweat', from Old High German sweiz, German Schwei, , Sanskrit svedah 'sweat', Greek hidros 'sweat', from Arabic sadria(t), Sadria(t) 'lit., a breast covering; a (Syrian) school uniform which usually covers the upper part of the body down to half-leg', from Sadr 'breast' via lexical shift, reordering, and turning /d/ into $/ \mathrm{t} /$.

Swims uit via Old English and Old High German swimman 'to move in or on water, float', German schwimmen, from Arabic $3 a w m$ 'to float, swim', turning /3/ into /s/; or zamma 'to be raised in water', turning /z/ into /s/. See suit.

Tailor (tail, Taylor) via Old French tailleur 'tailor; stone-mason; lit., a cutter', from Latin taliare 'to split', from talea 'a slender stick, rod; a cutting', direct from Arabic talla 'to pull', falla 'cut, unfurl', or Tawal 'to lengthen (robes)', turning /f (T)/ into /t/; however, more likely form Arabic dhail 'tail; edge of dress' via lexical shift and passing /dh/ into $/ \mathrm{t} /$; or dhaladhil, dhaldhal(at) 'what enters into a shirt's pocket' via lexical shift and merging /dh $\& \mathrm{dh} / \mathrm{into} / \mathrm{t} /$.

Tapestry (tapetum) via Old French, from Latin tappetium, from Greek tapes 'carpet, rug, heavy fabric', from Arabic bisaaT 'rug' via reordering and replacing /T/ by /t/.

Tattoo 'mark made on skin', direct from Arabic khuTooT 'lines' via lexical shift and merging /kh \& T/ into $/ \mathrm{t} /$.

Textile (text, texture) from Latin textilis 'a web, canvas, woven fabric, cloth', from texere (v) 'to weave', from Arabic takhyeeT 'stitching, sewing', khaaTa (v) via lexical shift and passing /kh $\& \mathrm{~T} /$ into /ks \& t/; or daqqa 'to sew; lit., to prick, sting', daqqaqa(t) (n) 'tailor' via lexical shift, changing /d/ into /t/, and /q/-split into /ks/.

Thread (throw) via Old English threed 'fine (twisted) cord', from Old High German drat, German Draht 'lit., twisted', from PIE root *tere- 'to rub (by turning), turning', direct from Arabic daar, dawra(t) (n) 'turn' via lexical shift and passing /d \& t/ into /th \& d/; or zarad/sard 'a (camel's neck) string; twisted ring; sewing' via lexical shift and passing /z (s)/ into /th/; or sidaara $(t)$ 'thread' via reordering and passing /s/ into /th/.

Tights (tighten, tight) via Old and Middle English thiht, thight, from Old Norse thettr 'solid', German dicht 'dense, tight', direct from Arabic Daiyeq(at) 'tight, narrow'; lexical shift and changing /D $\& \mathrm{q} /$ into /t \& gh/ applied.

Trousers from trouse via Gaelic or Middle Irish triubhas 'close-fitting shorts', direct from Arabic sitr, sutra $(t)$ 'a dress; cover' via reordering and lexical shift; or Tarz 'embroidery' or darz 'sewing' via lexical shift and passing $/ \mathrm{T} \& \mathrm{z} /$ into $/ \mathrm{t} \& \mathrm{~s} /$. 
Tuft (tuffet) via Old French touffe 'tuft of hair', from Latin tufa 'a kind of crest on a helmet', Greek toupha, or Old High German zopf, Old Norse toppr 'tuft, summit', from Arabic dhu'aaba(t) 'top; top, twisted hairs', turning /dh \& b/ into /t \& f/.

Tunic (tunicate) via Old English tunecce, from French, from Latin tunica 'undergarment', probably from Aramaic kittuna, direct from Arabic niTaaq 'girdle, belt' via lexical shift, reordering, and passing /T \& q/ into /t \& k/; Taqm 'a suit' via reordering and turning /T, q, \& m/ into /t, k, \& $\mathrm{n} /$; or tannoora $(t)$ 'skirt' via lexical shift and merging $/ \mathrm{r} \& \mathrm{t} /$ into $/ \mathrm{k} /$.

Twist (twizzle, twister) via Old English -twist 'divided object; rope; fork', from Proto-Germanic *twis-, from PIE *dwo- 'two', German zwist 'quarrel, discord', direct from Arabic Ta3waj(at), Ta3aja (v) 'twist, bend', turning /T, 3, \& j/ into /t, Ø, \& s/; or Tawia(t), Tawa (v.) 'folding', passing /T/ into /t/, and splitting /t/ into /st/ (cf. Tawsha(t) 'quarrel, discord', turning /T \& sh/ into $/ \mathrm{t} \& \mathrm{~s} /$ ).

For two, see Jassem (2012a; 2014g).

Underwear (wear and tear) is a compound of (i) under, via Old English under 'under, among', Latin infra, and Sanskrit adnah 'under', from Arabic 'adna 'lower', comparative of doon 'below, down' via reordering and /r/-insertion (Jassem 2014c) and (ii) wear below.

Veil (unveil, reveal) via Old French veil, from Latin vela 'veil, sail, curtain', from PIE root *weg- 'to weave a web', from Arabic lifaa3 'a veil' via reordering and /3/-loss (Jassem 2012b, 2014e); (cf. *weg- and Arabic $2 a a k$ 'to weave', turning $/ 2 \& \mathrm{k} /$ into /w \& g/; or wajjah 'to cover a quilt or mattress', passing /j/ into /g/).

Velvet (velveteen, velvety, velour, villus) probably via Old Provençal veluet, from Vulgar Latin *villutus 'velvet; lit., shaggy cloth', from villus 'shaggy hair, nap of cloth, tuft of hair', probably from velus 'fleece', from PIE root *wel- 'to tear, pull', from Arabic leef(at) 'fibre' or laffa(t), laflafa(t) 'a roll; a folding' via reordering and lexical shift; falla(t) 'unfurl, untie' or fatl(at) 'a thread; a twisting' via lexical shift and reordering; lahlah/nahnah, halhal 'soft, thin dress' via reordering and turning $/ \mathrm{h} /$ into $/ \mathrm{v} /$; or foof 'thin dress' via $/ / /$-insertion. See fleece.

Vest (vestment, vesture, invest) via Italian vesta, veste 'robe, gown', from Latin vestis 'clothing', from vestire (v) 'to clothe; put into possession, invest', from Arabic basT 'breast; happiness; spread (hand)' and related bisaaT 'a rug' or bazza(t) 'suit' via lexical shift and passing /b \& z (T)/ into $/ \mathrm{v} \& \mathrm{t} /$. See wear.

Vogue via Middle French vogue 'height of popularity or accepted fashion; fashion, success; drift; lit, a rowing', from Old French voguer 'to row, sway, set sail', direct from Arabic mawj 'wave; sway', fawj 'wave; division, group', or mufawwaq 'decorated and strapped dress', from fawq 'above; success', passing /m \& j (q)/ into /v \& g/.

Wardrobe (warden; guard) via Old English weard 'a guarding, protection; keeper, watchman', Old High German wart, from PIE root wer- 'to perceive' (cognates: Latin verere 'to observe with awe, fear', Greek ouros 'a guard', and possibly horan 'to see', French garder), straight from Arabic ra'a, ru'ia(t) (n) 'to see' via reordering; however, it comes direct from Arabic daar, door (pl.) 'room, house; turning' via reordering and lexical shift. See robe.

Wear (wear and tear) via Old English and Old High German werian 'to clothe, put on, cover up', from PIE *wos-eyo-, from root wes- 'to clothe' (cognates: Sanskrit vaste 'he puts on', Latin vestire 'to clothe', Greek esthes 'clothing'), straight from Arabic waara 'to hide, to wear' or hara 'to wear out', turning $/ \mathrm{h} /$ into $/ \mathrm{w} /$.

As to tear, it comes from Arabic tarra (Tarra) 'tear, cut' or farra 'unsew', turning /f/ into /t/; or dha'ar 'eye drop' where /dh/ became /t/ (Jassem 2013m).

Weave (weaver) via Old English wefan 'to weave', from German weben 'to weave', (Sanskrit ubhnati 'he laces together', Greek hyphe, hyphos 'web', Old English webb 'web'), from Arabic laffa/lawa 'to fold, tie', turning /// into /w/; or kubba(t), kabkab (v) 'to spin-roll' or kaffa 'of clothes, to tie back in', turning $/ \mathrm{k} \& \mathrm{~b} /$ into $/ \mathrm{w} \& \mathrm{v} /$. 
Wool (woolen, wooly) via Old English wull 'wool', from Proto-Germanic *wulna, (German wolle), from PIE *wele- 'wool', (Sanskrit urna, Greek lenos 'wool', Latin lana 'wool'), from Arabic 3ihn 'wool', merging $/ 3 \& \mathrm{~h} /$ into $/ \mathrm{w} /$ and $/ 1 \& \mathrm{n} /$ into $/ \mathrm{V}$; or hayoola 'cotton' via lexical shift and /h \& w/-merger.

Yarn via Old English gearn 'spun fibre or wool', from Proto-Germanic *garnan, (German garn), from PIE *ghere- 'intestine, gut, entrail', (Sanskrit hira 'vein; entrails', Greek khorde 'intestine', Latin hernia 'rupture'), from Arabic khirraan 'intestine', turning /kh/ into /g/; neer 'thread and spinning wood; yoke' via reordering; or qarn 'horn; connection; rope' via lexical shift and turning /q/ into /y/.

In short, the total number of fashion and modeling terms in this study amounted to 130, all of which have true Arabic cognates: i.e., $100 \%$.

\section{DisCUSSION}

Two issues merit further discussion, which concern the relevance of the results and their implications to (i) the radical linguistic theory and (ii) English and Indo-European etymological dictionaries and lexicographical works.

As to the former, fashion and modeling terms in Arabic, English, German, French, Latin, Greek, Sanskrit, and all Indo-European languages are, it can be clearly seen in the results, true cognates in having identical or similar forms and meanings, whose differences, however, are all due to natural and plausible causes and different routes of phonetic, morphological, grammatical, and semantic change. The percentage of shared fashion and modeling words between Arabic, English, Latin or Greek, for example, amounted to $100 \%$, which indicates their membership to or being dialects of the same language, for which, according to Cowley's (1997: 172-173) 100-word list-based classification, a 60$80 \%$ ratio is usually set.

Thus, the results fully agree with the findings of previous studies (Jassem 2012a-f, 2013a-q, 2014a-k, 2015a-j, 2016a-b) in which English, German, French, Latin, Greek, Sanskrit, and Arabic were all found to be not only members of the same family but also rather dialects of the same language. More precisely, they lend further support to the radical linguistic (or lexical root) theory on all theoretical and practical or procedural levels of analysis. Theoretically, the main principle which states that Arabic, English, German, French, and the so-called Indo-European languages are not only genetically related but also are dialects of the same language is, therefore, verifiably sound and empirically true. Because of this, a larger language family grouping has been proposed and termed Eurabian or Urban as a blend of Indo-European and Arabian languages (Jassem 2015c: 41, 2015d).

Moreover, the above linguistic scene entails that all the previous languages must have descended from an earlier, perfect, suddenly-emerged language, called radical (world) language from which all human languages initially came and which has variably survived into today's languages, though getting simpler and simpler over time. In other words, the radical language could never have died out beyond recognition. With proper methodology, it can be easily recovered as shown here (cf. Campbell 2004: 360). As this work demonstrated, it seems that its closest or most conservative and productive descendant is Arabic for having preserved almost all its features (Jassem 2014h-k, 2015a-d). In fact, all Indo-European languages can be safely aid to have descended directly from Arabic for reasons outlined earlier (Jassem 2015a-b, 2015d: 131-132; 2014a-b, 2014e).

As a consequence, reconstructing a first old world language, a proto-language, is needless; rather that proto-language, called radical language here, is still very much alive, having variably survived into today's languages, with Arabic being its closest descendant as the above data clearly shows (for detail, see Jassem 2014h: 254-256, 2014i: 116-117; 2014k, 2015a-b). Thus, instead of reconstructing hypothetical, fictitious languages, the quest should be concerned with relating those languages to it as shown here (cf. Campbell 2004: 360). Because the relationships amongst such languages are selfevident and because linguists deal with language first and foremost, the exact time and place of the split-up between Arabic and the so-called Indo-European languages is immaterial here (for details, see Jassem 2015e-f).

Not let's turn to the analytical level. It was clearly seen in the results above that the procedures of the theory operated neatly and smoothly on all plains. Phonetically, the entire changes were natural and plausible, cyclic and multi-directional, including processes like substitution, deletion, reversal, 
merger, split, reordering, reduction, and so on. The results are replete with examples which need not be repeated here.

Morphologically, the affixes, whether inflectional or derivational, had true Arabic cognates as well. For example, the commonest affixes and their variants in English and all Indo-European languages like (i) $-n$ and its variants (-an, -en, -ene, in-, -ine, -ing, -ness, -ar), (ii) - $t$ and its variants (-ate, -ette, ite, -ity; ad-, de-, -ed; -s, -ess, -ous, -ose), (iii) -tion, and (iv) -al and its variants (-eal, -ile, -elle) have true identical cognates in Arabic (for detail, see Jassem 2012f, 2013a-b, 20131, 2015d, 2016a).

Semantically, almost all types of lexical relations were attested. Lexical stability was a common pattern where many fashion and modeling terms preserved their basic meanings across the languages, e.g., attire, burnoose, cap, dress, gown, jacket, robe, wear. Lexical convergence was recurrent in the data due to formal and semantic similarity between Arabic words, on the one hand, and their English, German, French, Latin, and Greek cognates, on the other. In fact, all the words for which more than one likely cognate was provided are a case in point such as attire, band, boot, button, chemise, cloak, cloth, comb, coat, embroidery, robe, yarn, each of which might derive from two or more formally and semantically similar Arabic words (see 3 above). Although only one cognate might be the ultimate source in the end, no need is presently felt to specify which one it might be; the reader may judge. Likewise, semantic multiplicity (polysemy) was recurrent, where some English words had more than one meaning, which might just as well have more than one likely Arabic cognate; for example, boot, fabricate, fatigues, fit/outfit, guise (disguise), wear (and tear) have different meanings, every one of which derives from formally similar but semantically different Arabic words (see 3 above). As a matter of fact, almost all Arabic words are contextually polysemous in nature as can be immediately gleaned from any lexical entry (e.g., Ibn Manzour 2013). Lexical shift frequently occurred, e.g., band, blouse, bra, costume/custom, fashion, model, pajama, stitch, vogue. Lexical divergence was rare as in rip, from Arabic $r a$ 'ab 'to mend'; furl is another example (see 3. above). Lexical split might have affected several words such as short, shirt, skirt, all from Arabic qaSeer(at) 'short' via /q \& S/-merger into /sh/ and/or reordering (see 3. above); sleeve and slippers (slip) split from Arabic labisa 'to dress', costume and custom from Arabic kasm 'manner, body, shape'; net, knit, and nexus from Arabic nasj 'knitting'; hat and hood from Arabic 2aTTa 'hat'. Lexical change, which could be seen as an advanced stage of lexical shift, was rare such as fashion, model, stitch, and vogue. Finally, lexical variability occurred often in the data, whether at the level of the different forms of the same word within the same language such as English cloak, clock; gird, girth, girdle; high, heh, heah; or across the languages like English Jersey, Latin Caesarea, and Arabic jazeera(t) 'island' (see 3 above). Arabic, in particular, is replete with linguistic variability of all types such as fishshash(un) (fishfash, fashoosh) 'a coarse or soft dress', sa2na(t), su2na(t), sa2naa' 'facial skin or colour', Ta3aj, Ta3waj 'twist'; sandal, Sandal 'sandal', 3imma(t), 3amaama(t) 'helmet'; farw, farwa(t), firaa' 'fur'; halhal, lahlah, nahnah 'velvet; soft dress' (see 3 above).

Now, as regards English and Indo-European etymological dictionaries and their methodologies, it can be safely said that, although tracing the Arabic origins of English, German, French, Latin, Greek, and Sanskrit words cannot actually be carried out without referring to their etymologies such as Harper (2016) and his sources, yet they suffer from countless problems and drawbacks of different types. There are cases whose etymologies are not only admittedly uncertain or unknown but also are factually implausible, erroneous, and complicated with unnecessarily lengthy derivations and subderivations.

The most noticeable and serious shortcomings can be summed up as follows. The first concerns the nature of cognates. Since a cognate is defined as a word in two or more languages with the same or similar form and meaning, a great many such cognates, one can instantly observe, do not stand the test- i.e., they do not relate to each other in either form or meaning or both. For instance, the following sets:

blouse, barn and Provençal French (lano) blouso;

chemise and German hemd;

dress and Latin directus;

fatigues and Latin fatigare; 
fleece and Latin pluma;

fur and German Futter, and PIE * $p a$ - 'to feed, to protect';

furl and Latin firmus (+ ligare);

gaiter and German Rist;

guise and Old High German wisa and PIE weid-;

hair and Old High German kharan and PIE ghers-;

hat and Latin cassis;

heel and PIE kenk-;

jewel and Latin gaudium.

needle and Greek nein, Latin nere and PIE *(s)ne-;

pantaloon and Greek 'entirely lion; all compassionate';

robe/rope and German roub 'robbery';

seam/sew and Greek hymen 'thin skin' and hymnos 'song';

ring and Latin curvus 'bent, curved', crispus 'curly';

shirt/shorts and PIE *(s)ker-;

spin and Greek patos 'garment';

suit and Latin sequi 'to follow';

sweater and Greek hydros 'sweat';

veil and PIE *weg-;

vest and wear;

wear and Sanskrit vaste 'he puts on', Latin vestire 'to clothe', and Greek esthes 'clothing';

wool and Greek lenos 'wool', Latin lana 'wool', and Sanskrit urna 'wool'; yarn and Greek khorde 'intestine', and Latin hernia 'rupture', Sanskrit hira 'vein, intestine', and PIE *ghere- 'intestine, gut, entrails'; etc.

cannot all be cognates by form at all, under any circumstances; they are rather semantic or lexical equivalents just like any other words in different languages. All these words have Arabic cognates, though (see 3 above).

The second relates to unnecessarily lengthy derivation and sub-derivation with two or more parts as in costume (3 parts), cover (2 parts), dress, fabric, fashion, furl, glove, model, pullover, twist, and so on. Take glove, for instance, which is derived from (i) $g a$ - 'collective prefix' and (ii) lofi 'hand' in Latin, which does not make much sense, semantically-speaking. Its Arabic cognate(s) kaff 'palm of the hand' or ghilaaf 'cover' are self-evident as each agrees with it formally and semantically: i.e., a true cognate. Or take twist, which is traced back to Proto-German *twis-, from PIE * $d w o$ - 'two'; its Arabic cognate(s) are more direct and sensible.

The third is uncertain, unknown, or possible derivations. In fact, 21 out of $130(16 \%)$ such words occurred in the data like bikini, blouse, burnoose, cap, carpet, chemise, cuff, curly, fibre, fit, fleece, frill, furl, helmet, Jersey, jewel, pajama, velvet for which Arabic again readily provides the answer. Take blouse, for instance, which is said to be perhaps akin to Provençal (lano) blouso 'short (wool)', which does not make sense, formally and semantically speaking. Instead, deriving it from Arabic (i) libas 'clothing' preserves both (a) its form via reordering and (b) its meaning through lexical shift, both being clothes, or (ii) bazz 'clothes or such a type' via lexical shift, ///-insertion, and turning /z/ into /s/. Or compare chemise and German hemd 'shirt', the latter of which makes more sense if derived from Arabic hidm 'dress, clothing' via reordering and lexical shift (see 3 above). Or consider jewel, which came via Old French, perhaps from Latin jocale, from jocus 'pastime, sport', or from Latin 
gaudium 'rejoice', direct from Arabic jawhar(at) 'jewel' via /h/-loss and passing /r/ into /1/. Therefore, in all such cases, a direct derivation from Arabic is not only shorter but also more logical, which, at the same time, preserves both the form and meaning of cognate words. The same applies for the other instances as can be seen in the Results' Section 3 above.

The fourth issue concerns proto-language, whether proto-Germanic or PIE (proto-Indo-European). These hypothetical, fictitious languages have no linguistic status whatsoever, which constitute a spurious barrier between such languages and their immediate neighbours. Linking them to Arabic is not only a natural, geographical choice but also a linguistically logical, true, and substantive one. The data at hand attests to that, which speak better than words. Therefore, reconstructing PIE, for example, is futile, meaningless, and a waste of time and effort (see above).

Finally, there is the neglect of Arabic and its restriction to a few loans here and there, both deliberately and indeliberately. For instance, the number of such loans in this study was 3/130 $=0.23 \%$ - viz., cotton, jacket, mattress. As has already been seen in the results, all fashion and modeling terms have Arabic cognates as has been the case in all previous (47) studies, in fact. Why has this been so? This needs a separate portfolio, indeed. One only has to recall, though, that the modern European civilization and culture inherited Arabic science and culture in full through contact in Western Europe, especially Spain, France, and Italy.

\section{CONCLUSION AND RECOMMENDATIONS}

The main findings can be summed as follows:

- The 130 fashion and modeling terms in Arabic, English, German, French, Latin, Greek, and Sanskrit are true cognates, whose differences are due to natural and plausible causes and different routes of linguistic change.

- The radical linguistic (or lexical root) theory has been adequate for genetically relating fashion and modeling terms in the above languages to one another, according to which they are all dialects of the same language, comprising one larger language family grouping that may be called Eurabian or Urbant. In addition, these languages descended from a perfect Radical (Root) Language, or early prehistoric language, from which all human languages came and which, furthermore, has variably survived into today's languages. As Arabic is phonetically, morphologically, and lexically the most complex of all, it can be safely said that it has inherited almost all that Radical Language features, thus being the most conservative of all.

- On the analytical level, the main phonetic changes included substitution, reversal, reordering, deletion, split, and merger; morphologically, all affixes have true Arabic cognates; lexically, the recurrent patterns were stability, convergence, multiplicity, shift, split, and variability.

- Finally, further research is needed into all language levels, especially lexis (Jassem 2012a-f, 2013a-q, 2014a-k, 2015a-h, 2016b); it also calls for similar research in other world languages or their families (Jassem 2014h-i, 2015h-i, 2016a). Also the application of such findings to language teaching, lexicology and lexicography, translation (Jassem 2014d, 2015a), cultural (including historical, social, religious) awareness, understanding, and heritage is badly needed to promote cross-cultural cooperation and understanding.

\section{ACKNOWLEDGEMENTS}

Sincere thanks are warmly extended to everyone who contributed to this research in any way worldwide. For my supportive and inspiring wife, Amanie M. Ibrahim, I remain indebted as ever.

\section{REFERENCES}

Albabidi, Ahmad Mustafa Aldimashqi. (2011). Mu3jam asma' alashia' (allaTa'if fi allughat). Ed. Awad, Ahmad Abd-ul-tawab. Cairo: Dar AlfaDeelat. Retrieved http://www.waqfeya.com (March 1, 2016).

Algeo, J. (2010). The origins and development of the English language. (6th edn.). Wadsworth Cengage Learning. 
Altha3aalibi, Abu ManSoor. (2011). Fiqhu allughat wa asraar al3arabiyyat. Ed. by Alayoobi, Dr. Yaseen. Beirut and Saida: Al-Maktabat Al-3aSriyyat.

Bergs, Alexander and Brinton, Laurel (eds). (2012). Handbook of English historical linguistics. Berlin: Walter de Gruyter.

Campbell, L. (2006). Historical linguistics: An introduction. (2nd edn). Cambridge, Mass.: The MIT Press.

Celce-Murcia, M. et al. (2010). Teaching pronunciation: A course book and reference guide. $\left(2^{\text {nd }}\right.$ edn). Cambridge: Cambridge University Press.

Crowley, T. (1997). An Introduction to historical linguistics. (3rd edn). Oxford: Oxford University Press.

Crystal, D. (2010). The Cambridge encyclopedia of language. (3rd ed). Cambridge: Cambridge University Press.

Harper, Douglas. (2015). Online etymology dictionary. Retrieved http://www.etymonline.com (March 5, 2016).

Ibn Manzoor, Abi Alfadl Almisri. (2013). Lisan al3arab. Beirut: Dar Sadir. Retrieved http://www.lisan.com (March 5, 2016).

Ibn Seedah, Ali bin Ismail. (1996). AlmukhaSSaS. Beirut: Daar I2ya Alturath Al3arabi and Muassasat Altareekh alBarabi.

Jassem, Zaidan Ali. (1987). Phonological variation and change in immigrant speech: A sociolinguistic study of a 1967 Arab-Israeli war immigrant speech community in Damascus, Syria. PhD Thesis, Durham University, UK. Retrieved http://etheses.dur.ac.uk/1682/1/1682.pdf (March 5, 2016).

Jassem, Zaidan Ali. (1993). Dirasa fi 3ilmi allugha al-ijtima3i: Bahth lughawi Sauti ijtima3i fi allahajat al3arabia alshamia muqaranatan ma3a alingleeziyya wa ghairiha. Kuala Lumpur: Pustaka Antara. Retrieved http://www.academia.edu (March 5, 2016).

Jassem, Zaidan Ali. (1994a). Impact of the Arab-Israeli wars on language and social change in the Arab world: The case of Syrian Arabic. Kuala Lumpur: Pustaka Antara.

Jassem, Zaidan Ali. (1994b). Lectures in English and Arabic sociolinguistics, 2 Vols. Kuala Lumpur: Pustaka Antara.

Jassem, Zaidan Ali. (2012a). The Arabic origins of numeral words in English and European languages. International Journal of Linguistics 4 (3), 225-41. Retrieved URL: http://dx.doi.org/ 10.5296/ijl.v4i3.1276 (March 5, 2016).

Jassem, Zaidan Ali. (2012b). The Arabic origins of common religious terms in English: A lexical root theory approach. International Journal of Applied Linguistics and English Literature 1 (6), 5971. Retrieved URL: http://dx.doi.org/10.7575/ijalel.v.1n.6p.59 (March 5, 2016).

Jassem, Zaidan Ali. (2012c). The Arabic origins of English pronouns: A lexical root theory approach. International Journal of Linguistics 4 (4), 83-103. Retrieved URL: http://dx.doi.org/10.5296/ ijl.v4i4.227 (March 5, 2016).

Jassem, Zaidan Ali. (2012d). The Arabic origins of determiners in English and European languages: A lexical root theory approach. Language in India 12 (11), 323-359. Retrieved URL: http://www.languageinindia.com (March 5, 2016).

Jassem, Zaidan Ali. (2012e). The Arabic Origins of Verb "To Be" in English, German, and French: A Lexical Root Theory Approach. International Journal of Applied Linguistics and English Literature 1 (7), 185-196. Retrieved URL: http://dx.doi.org/10.7575/ijalel.v.1n.7p.185 (March 5, 2016).

Jassem, Zaidan Ali. (2012f). The Arabic origins of number and gender markers in English, German, French, and Latin: a lexical root theory approach. Language in India 12 (12), 89-119. Retrieved URL: http://www.languageinindia.com (March 5, 2016).

Jassem, Zaidan Ali. (2013a). The Arabic origins of derivational morphemes in English, German, and French: A lexical root theory approach. Language in India 13 (1), 48-72. Retrieved URL: http://www.languageinindia.com (March 5, 2016). 
The Arabic Origins of English and Indo-European "Fashion and Modeling Terms": A Radical Linguistic Theory Approach

Jassem, Zaidan Ali. (2013b). The Arabic origins of negative particles in English, German, and French: A lexical root theory approach. Language in India 13 (1), 234-48. Retrieved URL: http://www.languageinindia.com (March 5, 2016).

Jassem, Zaidan Ali. (2013c). The English, German, and French cognates of Arabic back consonants: A lexical root theory approach. International Journal of English and Education 2 (2): 108-128. Retrieved URL: http://www.ijee.org (March 5, 2016).

Jassem, Zaidan Ali. (2013d). The Arabic origins of "water and sea" terms in English, German, and French: A lexical root theory approach. Language in India 13 (2): 126-151. Retrieved URL: http://www.languageinindia.com (March 5, 2016).

Jassem, Zaidan Ali. (2013e). The Arabic origins of "air and fire" terms in English, German, and French: A lexical root theory approach. Language in India 13 (3): 631-651. Retrieved URL: http://www.languageinindia.com (March 5, 2016).

Jassem, Zaidan Ali. (2013f). The Arabic origins of "celestial and terrestrial" terms in English, German, and French: A lexical root theory approach. International Journal of English and Education 2 (2): 323-345. Retrieved URL: http://www.ijee.org (March 5, 2016).

Jassem, Zaidan Ali. (2013g). The Arabic origins of "animal" terms in English and European languages: A lexical root theory approach. Language in India 13 (4): 68-106. Retrieved URL: http://www.languageinindia.com (March 5, 2016).

Jassem, Zaidan Ali. (2013h). The Arabic origins of "body part" terms in English and European languages: A lexical root theory approach. International Journal of Current Applied Linguistics and English Literature (1). Retrieved URL: http://www.bretj.com (March 5, 2016).

Jassem, Zaidan Ali. (2013i). The Arabic origins of "speech and writing" terms in English and European languages: A lexical root theory approach. Language in India 13 (5): 108-159. Retrieved URL: http://www.languageinindia.com (March 5, 2016).

Jassem, Zaidan Ali. (2013j). The Arabic origins of "time words" in English and European languages: A lexical root theory approach. Language in India 13 (6): 274-97. Retrieved URL: http://www.languageinindia.com (March 5, 2016).

Jassem, Zaidan Ali. (2013k). The Arabic origins of "family words" in English and European languages: A lexical root theory approach. International Journal of English and Education 2 (3): 261-77. Retrieved URL: http//www.ijee.org (March 5, 2016).

Jassem, Zaidan Ali. (20131). al'uSool abarabiat lilDamaa'ir alshakSiat fi alingleeziat, walfiransiat, walalmaniat (The Arabic origins of "personal pronouns" in English, German, and French: A lexical root theory approach (In Arabic). Almu'tamar aldawli althamin, tajdeed alkhiTaab al3arabi, jaami3at imam bonjul, Indonesia 28-31 August 2013 ( $8^{\text {th }}$ International Conference of Arabic Speech Renewal, Imam Bonjul University, Indonesia, 28-31 August 2013). Retrieved URL: http://www.academia.edu (March 5, 2016).

Jassem, Zaidan Ali. (2013m). The Arabic origins of "cutting and breaking words" in English and European languages: A lexical root theory approach. Research Journal of English Language and Literature 1 (2): 155-68. Retrieved URL: http//rjelal.com (March 5, 2016).

Jassem, Zaidan Ali. (2013n). The Arabic origins of "movement and action words" in English and European languages: A lexical root theory approach. Research Journal of English Language and Literature 1 (3): 187-202. Retrieved URL: http://rjelal.com (March 5, 2016).

Jassem, Zaidan Ali. (2013o). The Arabic origins of "perceptual and sensual words" in English and European languages: A lexical root theory approach. Research Journal of English Language and Literature 1 (4): 212-24. Retrieved URL: http://rjelal.com (March 5, 2016).

Jassem, Zaidan Ali. (2013p). The Arabic origins of "cognitive and mental words" in English and European languages: A lexical root theory approach. International Journal of English and Education 2 (4): 65-83. Retrieved URL: http//www.ijee.org (March 5, 2016).

Jassem, Zaidan Ali. (2013q). The Arabic origins of "love and sexual words" in English and European languages: A lexical root theory approach. International Journal of Language and Linguistics 1 (4): 97-114. Retrieved URL: http://www.ijll.org (March 5, 2016). 
Jassem, Zaidan Ali. (2014a). The Arabic origins of "wining and dining words" in English and European languages: A lexical root theory approach. International Journal of English and Education 1 (4): 146-74. Retrieved URL: http://www.ijee.org (March 5, 2016).

Jassem, Zaidan Ali. (2014b). The Arabic origins of "question and auxiliary words" in English and European languages: A lexical root theory approach. International Journal of Language and Linguistics 2 (1). Retrieved URL: http://www.ijll.org (March 5, 2016).

Jassem, Zaidan Ali. (2014c). The Arabic origins of "prepositions and conjunctions" in English and European languages: A lexical root theory approach. Journal for the Study of English Linguistics 2 (1). Retrieved URL: http://www.jsel.org (March 5, 2016).

Jassem, Zaidan Ali. (2014d). Translating cultural universals radically: A lexical root theory approach for translating English, French, and German cultural terms into Arabic. In لترجمة وإثكالات الثثاقفة بحوث وششاركات المؤتدر Proceedings of the International Conference on Translation and the Problematics of Cross-Cultural Understanding, the Forumfor Arab and International Relations, Doha, Qatar 26-27 February 2014: 643-695, 505-60).

Jassem, Zaidan Ali. (2014e). The Arabic origins of "divine and theological terms" in English, German, and French: A lexical root theory approach. Language in India 14 (3): 155-195. Retrieved URL: http://www.languageinindia.com (March 5, 2016).

Jassem, Zaidan Ali. (2014f). The Arabic origins of "proper names" in English and European languages: A lexical root theory approach. Research Journal of ELT and Poetry 2 (2): 201-22. Retrieved URL: http://www.journalofelt.in (March 5, 2016).

Jassem, Zaidan Ali. (2014g). The Arabic origins of "mathematical and computational terms" in English and European languages: A lexical root theory approach. International Journal on Studies in English and Literature 2 (5): 21-40. Retrieved URL: http://www.arcjournals.org/ijsell (March 5, 2016).

Jassem, Zaidan Ali. (2014h). The Arabic origins of "Mandarin Chinese Pronouns": A radical linguistic theory approach. International Journal of English and Education 3 (3). Retrieved URL: http://www.ijee.org (March 5, 2016).

Jassem, Zaidan Ali. (2014i). The Arabic origins of "Finnish and Basque Pronouns": A radical linguistic theory approach. Journal of English language and literature 2 (1): 109-20. Retrieved URL: http://www.jellonline.com (March 5, 2016).

Jassem, Zaidan Ali. (2014j). The Arabic origins of English and Indo-European "colour and artistic terms": A radical linguistic theory approach. International Journal of English language, literature, and Translation 1 (1): 1-14. Retrieved URL: http://www.ijels.com (March 5, 2016).

Jassem, Zaidan Ali. (2014k). I buy, Ich kaufe, \& J'achéte as Arabic Dialectal Variants: A radical linguistic theory approach. International Journal of language and linguistics 2 (5): 317-27. Retrieved URL: http://www.sciencepublishinggroup.com/ijll. Doi: 10.11648/j.ijll.20140205.15 (March 5, 2016).

Jassem, Zaidan Ali. (2015a). The Arabic origins of English and Indo-European "life and death terms": A radical linguistic theory approach. International Journal of English and Education 4/1: 322345. Retrieved URL: http//www.ijee.org (March 5, 2016).

Jassem, Zaidan Ali. (2015b). Towards a radical translation theory for names: A comparative historical linguistics approach. International Journal of English and Education 4/1: 298-321. Retrieved URL: http://www.ijee.org (March 5, 2016).

Jassem, Zaidan Ali. (2015c). The Arabic origins of English and Indo-European "medical terms": A radical linguistic theory approach. Journal of English Language and Literature 2/1: 18-47. Retrieved URL: http://www.joell.in (March 5, 2016).

Jassem, Zaidan Ali. (2015d). The Arabic origins or cognates of English and Indo-European "case markings and word order": A radical linguistic theory approach. Language in India 15/3: 104-40. Retrieved URL: http://www.languageinindia.com (March 5, 2016).

Jassem, Zaidan Ali. (2015e). The Arabic origins of English and Indo-European "democratic terms": A radical linguistic theory approach. Journal of English Language and Literature 2/2: 111-139. Retrieved URL: http://www.joell.in (March 5, 2016).

Jassem, Zaidan Ali. (2015f). The Arabic origins of English and Indo-European "military terms": A radical linguistic theory approach. Language in India 15/5: 105-139. Retrieved URL: http://www.languageinindia.com (March 5, 2016). 
The Arabic Origins of English and Indo-European "Fashion and Modeling Terms": A Radical Linguistic Theory Approach

Jassem, Zaidan Ali. (2015g). The Arabic origins of English and Indo-European "legal terms": A radical linguistic theory approach. Journal of Applied Linguistics and Translation 1/1: 10-29. Retrieved URL: http://www.languageinindia.com (March 5, 2016).

Jassem, Zaidan Ali. (2015h). allugha alarabia wa allugha alBalamia aljadhria alwaritha al2afiDha: asmaa' alishara fi lughat al3alam qadiman wa2adithan min manDhoor 3ilm allugha aljadhri (In Arabic, Arabic as the conservative radical world language: A radical linguistic theory approach to demonstratives in old and modern world languages). Proceedings of almultaqa al3ilmi al3aalami altaasi3 lilughati alarabia wa-l-mu'tamar alkhamis li-itti2ad mu3allimi allugha alarabia, Mawlana Malik Ibrahim Islamic University, Malang, Eastern Java, Indonesia, August 27-29, 2015.

Jassem, Zaidan Ali. (2015i). Negation in world languages: A radical linguistic theory approach. Veda's Journal of English Language and Literature 2/4: 1-17. Retrieved URL: http://www.joell.in (March 5, 2016)

Jassem, Zaidan Ali. (2016a). The Arabic origins of "plural markers" in world languages: A radical linguistic theory approach. International Journal of English and Education 5/1: 193-223. Retrieved URL: http://www.jjee.org (March 5, 2016). (Also in Indonesian EFL Journal 1 (2): 144-163, 2015.)

Jassem, Zaidan Ali. (2016b). The Arabic origins of English and Indo-European "floral terms": A radical linguistic theory approach. International Journal on Studies in English and Literature 4 (2): 81-99. Retrieved URL: http://www.arcjournals.org/ijsell (March 5, 2016).

Mu3jam alama3ani (2016). Retrieved URL: http://www.almaany.com (March 5, 2016).

Pyles, T. and J. Algeo. (1993). The origins and development of the English language. (4th edn). San Diego: HBJ.

Roach, P. (2008). English phonetics and phonology: A practical course. (4 ${ }^{\text {th }}$ edn). Cambridge: Cambridge University Press.

Yule, G. (2014). The study of language. ( $5^{\text {th }}$ ed). Cambridge: Cambridge University Press.

\section{AUTHOR'S BIOGRAPHY}

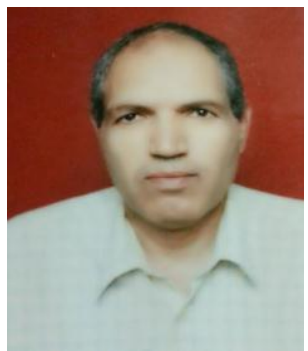

Zaidan Ali Jassem, currently Professor of English Language and Linguistics, Department of English Language, Linguistics and Translation, Qassim University, KSA. I obtained my $\mathrm{PhD}$ in Linguistics from Durham University, England, in 1987; M.A. in Applied Linguistics (TEFL) from the same institution in 1984; PG Diploma in English Literature from Damascus University, 1979; and B.A. in English Language and Literature from the same institution in 1978. I taught at several universities in Syria, Malaysia, and UK. I participated in many national and international conferences. My current research interests are Radical Linguistic Theory and its applications to language sciences. I write and publish profusely on the subject. 